\title{
Comparative study on the usefulness of Gentamycin-containing collagen implants in the treatment of patients with osteitis and osteomyelitis of the craniofacial skeleton
}

\author{
Paweł J. Zawadzki ${ }^{1}$, Konrad Perkowski², Michał Kotlarski' ${ }^{1}$, Anna Pietruczuk-Padzik ${ }^{3}$, \\ Lidia Chomicz ${ }^{4}$ \\ 1 Clinic of Cranio-Maxillo-Facial and Oral Surgery and Implantology, Medical University of Warsaw, Warsaw, Poland \\ 2 Department of Orthodontics, Medical University of Warsaw, Warsaw, Poland \\ ${ }^{3}$ Department of Pharmaceutical Microbiology, Medical University of Warsaw, Warsaw, Poland \\ ${ }^{4}$ Department of Medical Biology, Medical University of Warsaw, Warsaw, Poland
}

Zawadzki PJ, Perkowski K, Kotlarski M, Pietruczuk-Padzik A, Chomicz L. Comparative study on the usefulness of Gentamycin-containing collagen implants in the treatment of patients with osteitis and osteomyelitis of the craniofacial skeleton. Ann Agric Environ Med. 2017; 24(2): 299-302 doi: 10.5604/12321966.1235182

\begin{abstract}
I Abstract
Introduction and objective. A reduction in incidences of peri-surgical complications due to infections is achieved by antibiotic prophylaxis The objective of the study was to assess the usefulness of gentamycin-containing collagen implants (GCCI) in the treatment of patients with osteitis and osteomyelitis of the craniofacial skeleton.

Materials and method. The retrospective study included 103 patients with osteitis and osteomyelitis. 54 patients were treated intra-operatively with GCCI (Garamycin, EusaPharma, Europe). 49 patients were treated according to standard procedures. Light microscopy and in vitro culture techniques were applied for bacteria specific identification, and to investigate the resistance of detected microbiota to antibiotics. Patients received one dose of antibiotic pre-operatively. Post-operative antibiotic treatment was administered individually, according to clinical course and microbiological tests. The patients were followed-up on days 3, 7 and 14 after discharge for local complications; radiographic follow-up was performed 3,6 and 12 months after surgery.

Results. The course of post-operative antibiotic therapy was shorter in GCCl patients than in the control group (median 1 vs. 7 days); they also required shorter hospitalization (median 3 vs. 4 days). Implantation of GCCI significantly reduced the incidence of local complications ( $\mathrm{OR} 0.30,95 \% \mathrm{Cl} 0.11-0.83, \mathrm{p}<0.0001)$, independently of the use of postoperative antibiotic therapy. On follow-up after 3-12 months, all patients presented with good soft tissue and bone healing.

Conclusions. The results of this comparative study advocate the use of GCCl in osteomyelitis of various origin in oral and maxillofacial surgery, as they seemed to reduce the incidence of local complications, shorten antibiotic administration time and hospital stay.
\end{abstract}

I Key words

comparative study, Gentamycin-containing collagen treatment patients, osteomyelitis, craniofacial skeleton

\section{INTRODUCTION}

It is considered that inflammatory processes in the bones of the craniofacial skeleton constitute a serious therapeutic challenge in maxillofacial surgery. The histology of osseous tissue and its poor vascularisation result in low penetration of antibiotics administered systemically [1], necessitating high dosage and prolonged treatment. Osteitis and osteomyelitis of the jaws may originate from odontogenic infections, may be complications of trauma, due to exposure of the fracture gap to oral microbiota, or constitute long-term consequences of irradiation.

Gentamycin-containing collagen implants (GCCI) have been successfully used in the prophylaxis and treatment of surgical site infections in vascular [2], gastrointestinal [3], and cardiac surgery [4]. They have also been shown to play a role in the treatment of both acute and chronic osteomyelitis

Address for correspondence: Paweł J.Zawadzki, Clinic of Cranio-Maxillo-Facial and Oral Surgery and Implantology, Medical University of Warsaw, Poland, Lindleya 4. 02-005, Warsaw, Poland

e-mail: pawel.j.zawadzki@gmail.com

Received: 27 January 2017; accepted: 1 March 2017; first published on May 2017 in orthopaedic surgery $[5,6]$. Very limited data are presently available concerning the role of GCCI in the field of oral and maxillofacial surgery.

\section{OBJECTIVE}

The objective of this comparative study was to evaluate the usefulness of Gentamycin-containing collagen implants in the treatment of patients with osteitis and osteomyelitis of the craniofacial skeleton.

\section{MATERIALS AND METHOD}

103 patients aged 22-86 years, with osteitis and osteomyelitis, were included in the retrospective study. 54 patients treated in 2011-2013 underwent operations for various indications: posttraumatic osteomyelitis (8/54), extractions of impacted teeth (11/54), periapical granuloma, cyst and tumour removal (19/54), debridement of bone sequestra following odontogenic osteomyelitis (9/54; Fig. 1 and Fig. 2), and 


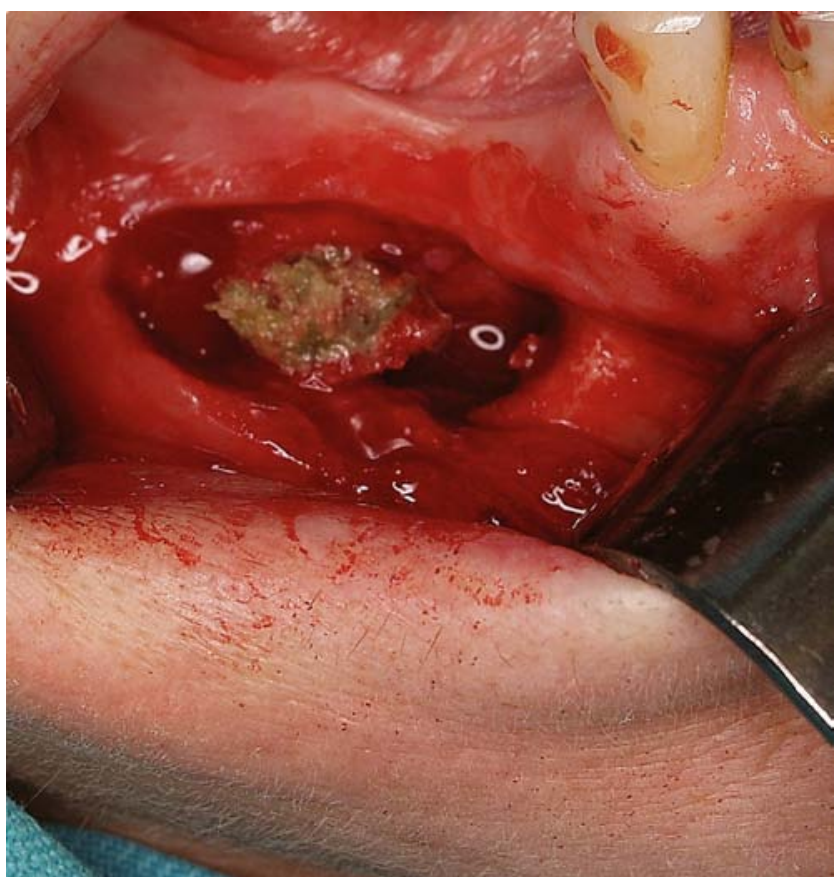

Figure 1. Odontogenic osteomyelitis of mandible - intraoperative image of sequestrum

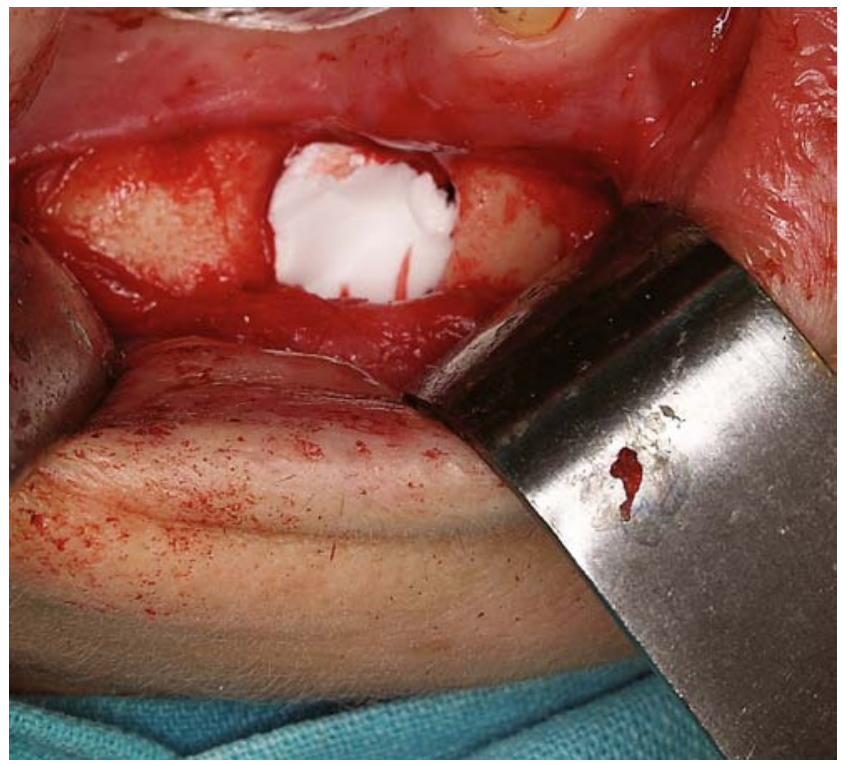

Figure 2. Filling of bone defect with Genatmycin sponge

osteoradionecrosis (7/54). Bone defects in the patients were filled intra-operatively with GCCI (Garamycin, EusaPharma, Europe). All procedures were performed according to unified protocols for each procedure type. A collagenous drug carrier loaded with Gentamycin was used strictly according to the manufacturer's instructions. These patients were compared to the control group of 49 patients treated for osteomyelitis in the same centre in 2009-2010, according to standard procedures.

Samples from some patients collected as swabs, secretions or oral tissue fragments were examined microbiologically. Wet and Giemsa-stained smears prepared before treatment for the preliminary identification of Gram-positive and Gram-negative bacteria strains under a light microscope, and conventional in vitro culture techniques, were applied for bacteria species identification. Chapman's plate growth medium for recovery and isolation of Staphylococcus strains and McConkey's medium for identification of Enterobacteriaceae were applied. Antibiotic- resistance tests of the identified microbiota were performed by standard in vitro techniques [7].

Patients of both groups received antibiotic pre-operatively. Post-operative antibiotic treatment was administered individually, according to clinical evaluation and microbiological tests.

Follow-up was conducted according to standard procedures adopted in our centre, which include clinical evaluation in our outpatient clinic on days 3, 7 and 14 after discharge, as well as radiologic follow-up performed 3, 6 and 12 months after surgery.

Statistical analysis Nominal variables were given as numbers with appropriate percentage whereas continuous variables as medians with interquartile ranges. Chi Square test with Yate's correction, Fisher's exact test and Freeman-Halton extension were used to test the associations. Continuous variables were analyzed using the Mann-Whitney's U test. Logistic regression was used for analysis of factors associated with the local complication development. A p value $<0.05$ was considered as statistically significant. Statistical analysis was performed with usage of STATISTICA 10.0 software (Statsoft, Tulsa, OK, USA).

\section{RESULTS}

The 2 patient groups were similar with regard to clinical indications for surgical procedure $(\mathrm{p}=0.45)$. They did not differ with regard to gender, presence of cardiovascular diseases, or factors influencing wound healing, such as diabetes mellitus, corticosteroid or hormone therapy. A similar number of patients in both groups received pre-and post-operative antibiotic therapy (Tab. 1), while thea period of antibiotic application was different and varied from 1-7 days.

Table 1. Characteristics of groups with regard to clinical indications for the surgical procedure

\begin{tabular}{lccc}
\hline & $\begin{array}{c}\text { GCCl group } \\
\text { N/total N, (\%) }\end{array}$ & $\begin{array}{c}\text { Control group } \\
\text { N/total N, (\%) }\end{array}$ & p value \\
\hline Sex: male & $25 / 54(46.30 \%)$ & $25 / 49(51.02 \%)$ & 0.6319 \\
\hline Cardiovascular diseases & $19 / 54(35.19 \%)$ & $16 / 49(32.65 \%)$ & 0.7864 \\
\hline $\begin{array}{l}\text { Factors influencing wound healing } \\
\text { (diabetes mellitus, corticosteroids, }\end{array}$ & $19 / 54(35.19 \%)$ & $21 / 49(42.86 \%)$ & 0.4250 \\
$\begin{array}{l}\text { hormone therapy) } \\
\text { Pre-operative antibiotic treatment }\end{array}$ & $46 / 54(85.19 \%)$ & $47 / 49(95.92 \%)$ & 0.0963 \\
\hline Post-operative antibiotic treatment & $38 / 54(70.37 \%)$ & $42 / 49(85.71 \%)$ & 0.1030 \\
\hline
\end{tabular}

$\mathrm{N}$ - number of patients

In the oral cavities of all patients, Gram- positive bacteria of the Streptococcus viridans group - typical inhabitants of the human oral cavity - were identified. Apart from the resident species, some opportunistic and pathogenic bacteria strains were detected. Among Gram- positive Staphylococci, the Staphylococcus epidermidis and Staphylococcus epidermidis MRCNS were identified. Enterobacter spp., Pantoea agglomerans were detected of Gram- negative Enterobacteriaceae. 
Patients in the GCCI group were significantly older (median: 51.5, 25\%-75\%.36.0-64.0 vs. $33.0,25 \%-75 \% 26.0-$ 47.0; p $<0.0001$ ) (Fig. 3). The use of GCCI influenced the administration of post-operative antibiotics: patients from the GCCI group received post-operative antibiotic therapy for a significantly shorter time than the control group (Fig. 4). Some patients did not receive antibiotics post-operatively, but most of the patients received antibiotic for 1-2 days; the antibiotic therapy was applied only occasionally for 7 days (median 1 day, 25\%-75\%: 0 -2.0 vs 7 days, 25\%-75\%:7.0-7.0; $\mathrm{p}<0.0001$ ) (Fig. 4). Also, the overall length of hospitalization depended on the use of GCCI, as patients from the GCCI group required fewer days of hospitalization (median 3.0, 25\%-75\%: $0.0-3.0)$ than patients from the control group (median 4.0, 25\%-75\%: 1.0-6.0; $\mathrm{p}=0.0003$ ) (Fig. 5).

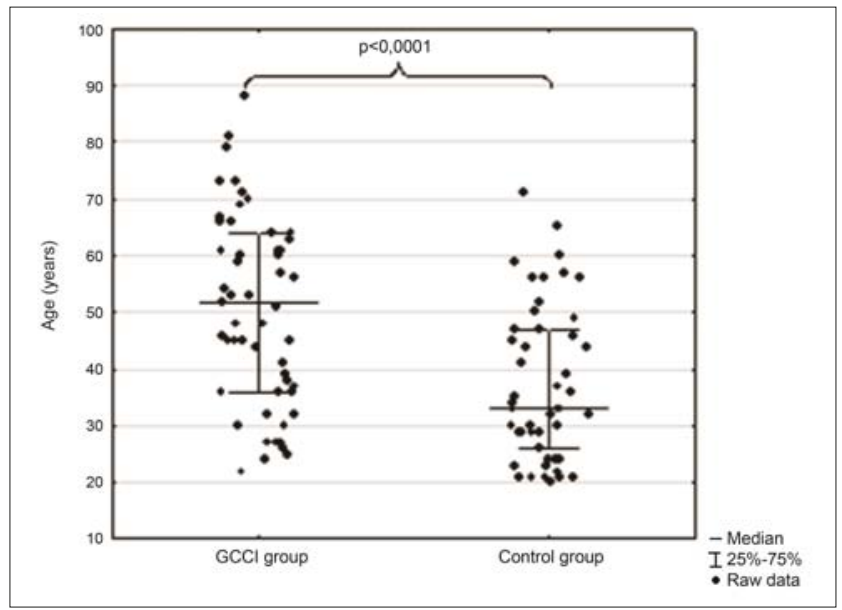

Figure 3. Age difference between $\mathrm{GCCl}$ group and control group

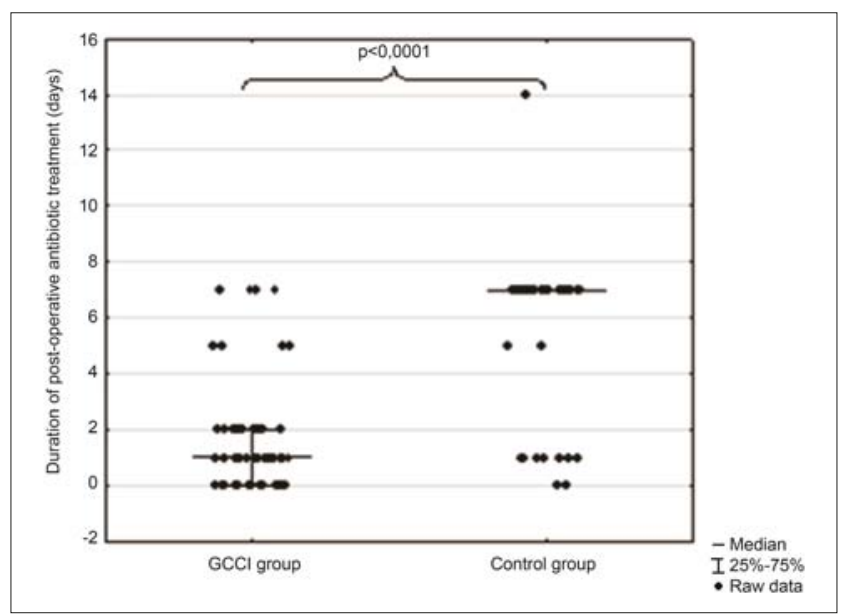

Figure 4. Difference in days of post-operative antibiotic treatment between groups

The incidence of local complications, described as wound dehiscence requiring secondary closure, differed according to the site of the procedure: they were significantly more frequently observed in the mandibula than in the maxillary region $(\mathrm{p}=0.02)$. They were observed more frequently in the patients of the control group than in GCCI group $(22 / 49,44.9 \%$ vs $14 / 54,25.9 \%)$, but did not reach statistical significance $(\mathrm{p}=0.07)$. In multivariate analysis, however, lack of GCCI use and older age were shown to be independent risk factors for local complication development. Implantation of GCCI significantly reduced the incidence of local complications

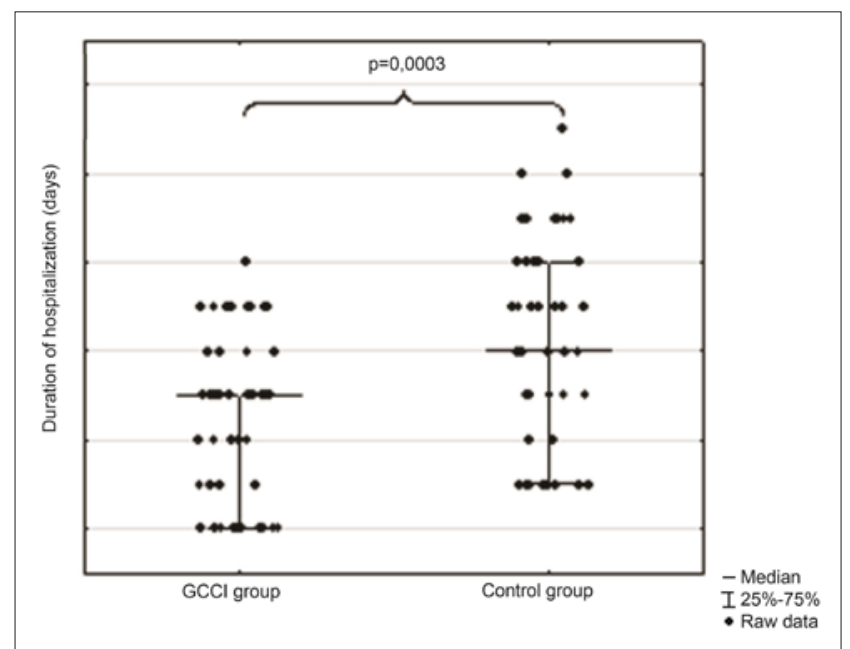

Figure 5. Difference in length of hospital stay between groups

(OR $0.30,95 \%$ CI $0.11-0.83$ ), independently of the use of postoperative antibiotic therapy. Local complications were shown to be more frequent in older patients (OR 1.04, 95\%CI $1.00-1.07 ; \mathrm{p}<0.0001)$.

Of note, the spongious structure of GCCI provided support for soft tissues in reconstructive procedures, especially in the treatment of post-surgical complications of fractures. Due to the nature of the collagenous carrier, better secondary healing by granulation in the case of wound edge dehiscence was observed.

On follow-up after 3-12 months, all patients presented with good soft tissue and bone healing.

\section{DISCUSSION}

The primary function of Gentamycin-containing collagen implants is to provide haemostasis [8,9]; however, a considerable number of studies have shown their usefulness in diminishing incidences of infectious complications in a wide variety of surgical applications. GCCI have been successfully used in reducing wound infection following vascular surgery, especially in high risk patients and procedures [10]. The adjuvant therapy with collagenous Gentamycin in combination with surgical debridement gave excellent results in the treatment of deep sternal wound infections [11]. In the presented study, GCCI implantation enabled shorter systemic antibiotic therapy and quicker hospital discharge. These results are similar to those in other fields, especially in gastrointestinal surgery, where GCCI proved to reduce the length of hospital stay by $40 \%$ [12]. The benefit was more pronounced in high infection risk procedures. Also, in osteomyelitis in orthopaedics, GCCI seemed to improve the outcome, as the duration of postoperative treatment was reduced by 16 days in the GCCI group, compared with patients in whom polymethylmethacrylate (PMMA) beads were used [13].

The continuously evolving bacterial resistance represents a great challenge in the treatment of osteomyelitis. Literature data enabling the proper choice of antibiotic therapy for chronic osteomyelitis is scarce; furthermore, the majority of published trials were conducted more than 20 years ago [14]. Prolonged systemic use of antibiotics can induce both antibiotic resistance and toxicity. Topical application of Gentamycin in a collagenous carrier may help solve this issue 
by reducing serum concentrations of the antibiotic, and thus reducing the risks of adverse events, while providing high local concentrations. It is well-established that the bactericidal effect of Gentamycin depends on the peak level, and a high peak level is associated with a high bactericidal effect [15, 16]. There is also evidence suggesting that the first dose of aminoglycoside is the most important in the course of therapy [17], and that the first dose of aminoglycoside has the most bactericidal effect on the bacterial population [18]. It has also been reported that attainment of a pharmacodynamic target of $C_{\max } / \mathrm{MIC} \geq 10$ within 48 hours of therapy is associated with a good therapeutic response [19]. Indeed, Gentamycin release from collagenous carrier ensures local antibiotic concentrations above minimal inhibitory concentrations (MIC) for at least 48 hours [20]. This may, at least partly, explain the successful reduction in the length of antibiotic therapy in the patients in the current srtudy. Gram- positive Staphylococcus epidermidis MSRA (-methycillin-resistant strains) detected in these patients may be a causative agent of gingivitis and endodontic infections responsible for treatment difficulties.

Gram-negative Enterobacteriaceae strains are known as causative agents of serious infections particularly dangerous for immunocompromised and elderly persons. Recently, the interrelations between the oral microbiome organisms associations with various local/general deteriorations and diseases have been an important objective of studies. Particularly, the role of biofilm with regard to interrelations between microbiota components and mouth tissues is emphasized. This complex structure directly affecting oral cavity tissues indicates a higher resistance to antimicrobial agents than free mouth microorganisms not embedded in biofilm. The findings confirmed the literature and own data regarding patients with oral cancer and other deteriorations [21-22].

Furthermore, in addition to its antibacterial effect, Gentamycin was suggested to promote early vascularization in the bone tissue [23]. In a relatively large group of patients with a traumatic tooth extraction with socket seal surgery, local application of Gentamycin promoted more vascular in growth in the blood clot and granulation tissue beneath the graft, thereby supplying better nourishment during the initial healing phase [24]. Both the anti-bacterial and pro-angiogenic roles of Gentamycin was suggested to speed bone formation following tooth extraction for as much as 2 weeks [24]. In another study, impregnation of a spongious bone substitute with Gentamycin tended to improve healing outcome after guided tissue regeneration [25].

\section{CONCLUSIONS}

Results of this study advocate the use of Gentamycincontaining collagen implants in osteomyelitis of various origin in oral and maxillofacial surgery. They provide good local results improving haemostasis and wound healing, and at the same time reduce the incidence of local complications independently of systemic antibiotic treatment. They may also help reduce adverse events of systemic antibiotic treatment by shortening antibiotic administration time. Finally, the local implantation of GCCI reduces the length of hospitalization after surgery. Validation of these results in prospective, randomized trials is mandatory.

\section{REFERENCES}

1. Goda A, Maruyama F, Michi Y, Nakagawa I, Harada K. Analysis of the factors affecting the formation of the microbiome associated with chronic osteomyelitis of the jaw. Clin Microbiol Infect 2014; 20(5): 0309-0317.

2. Hussain ST. Local application of gentamicin-containing collagen implant in the prophylaxis and treatment of surgical site infection following vascular surgery. Int J Surg. 2012; 10: S5-S9.

3. de Bruin AF, Gosselink MP, van der Harst E. Local application of gentamicin-containing collagen implant in the prophylaxis of surgical site infection following gastrointestinal surgery. Int J Surg. 2012; 10: S21-S27.

4. Raja SG. Local application of gentamicin-containing collagen implant in the prophylaxis and treatment of surgical site infection following cardiac surgery. Int J Surg. 2012; 10: S10-S14.

5. Friberg O, Svedjeholm R, Soderquist B, Granfeldt H, Vikerfors T, Kallman J. Local gentamicin reduces sternal wound infections after cardiac surgery: a randomized controlled trial. Ann Thorac Surg. 2005; 79: 153-161.

6. Knaepler H. Local application of gentamicin-containing collagen implant in the prophylaxis and treatment of surgical site infection in orthopaedic surgery. Int J Surg. 2012; 10: S15-S20.

7. Murray PR, Baron EJ, Jorgensen JH, Landry ML, Pfaller MA. Manual of clinical microbiology. 9th edition. ASM Press, Washington, USA, 2007.

8. Lovering AM, Sunderland J. Impact of soaking gentamicin-containing collagen implants on potential antimicrobial efficacy. Int J Surg.2012; 10: S2-S4.

9. Parker SJ, Brown D, Hill PF, Watkins PE. Fibrinogen-impregnated collagen as a combined haemostatic agent and antibiotic delivery system in a porcine model of splenic trauma. Eur J Surg. 1999; 165: 609-614.

10. Hussain ST. Local application of gentamicin-containing collagen implant in the prophylaxis and treatment of surgical site infection following vascular surgery. Int J Surg. 2012; 10: S5-S9.

11. Leyh RG, Bartels C, Sievers HH. Adjuvant treatment of deep sternal wound infection with collagenous gentamycin. Ann Thorac Surg. 1999; 68: 1648-1651..

12. de Bruin AF, Gosselink MP, Wijffels NA, Coene PP, van der Harst E: Local gentamicin reduces perineal wound infection after radiotherapy and abdominoperineal resection. Tech Coloproctol. 2008; 12: 303-307.

13. Attmanspacher W, Dittrich V, Schatzler A, Stedtfeld HW: [Mid-term outcome of postoperative infections of the shoulder]. Unfallchirurg. 2000; 10: 1048-056

14. Conterno LO, Turchi MD. Antibiotics for treating chronic osteomyelitis in adults. Cochrane Database Syst Rev 2013; 6 (9):CD004439. doi: 10.1002/14651858.CD004439.pub3.

15. Moore RD, Smith CR, Lietman PS: The association of aminoglycoside plasma levels with mortality in patients with gram-negative bacteremia. J Infect Dis. 1984; 149: 443-448.

16. Moore RD, Lietman PS, Smith CR: Clinical response to aminoglycoside therapy: importance of the ratio of peak concentration to minimal inhibitory concentration. J Infect Dis. 1987; 155: 93-99.

17. Xiong YQ, Caillon J, Kergueris MF, Drugeon H, Baron D, Potel G, Bayer AS: Adaptive resistance of Pseudomonas aeruginosa induced by aminoglycosides and killing kinetics in a rabbit endocarditis model. Antimicrob Agents Chemother 1997; 4: 823-826.

18. Tam VH, Kabbara S, Vo G, Schilling AN, Coyle EA: Comparative pharmacodynamics of gentamicin against Staphylococcus aureus and Pseudomonas aeruginosa. Antimicrob Agents Chemother. 2006; 50: 2626-2631.

19. Kashuba AD, Bertino JS, Jr., Nafziger AN: Dosing of aminoglycosides to rapidly attain pharmacodynamic goals and hasten therapeutic response by using individualized pharmacokinetic monitoring of patients with pneumonia caused by gram-negative organisms. Antimicrob Agents Chemother. 1998; 42: 1842- 1844.

20. Rutten HJ: Local application of gentamycin-containing collagen implant in the prophylaxis and treatment of surgical site infection. Int J Surg. 2012; 10: S1.

21. Wróblewska M, Strużycka I, Mierzwińska-Nastalska E. Significance of biofilms in dentistry. Przegl. Epidemiol. (Epidemiological Review). 2015; 69: 739-744.

22. Zawadzki PJ, Starościak B, Perkowski K, Baltaza W, Padzik M, Pionkowski K, Chomicz L. et al. Comparative assessment of selected intraoral microorganisms - potential factors for peri-surgical management complications. Annals of Parasit. 2016; 62(3): 233-237.

23. Holck DE, Dutton JJ, Proia A, Khawly J, Mittra R, Dev S, Imami N. Rate of vascularization of coralline hydroxyapatite spherical implants pretreated with saline/gentamicin, rTGF-beta 2, and autogenous plasma. Ophthal Plast Reconstr Surg. 1998; 14: 73-80.

24. Oghli AA, Steveling H: Ridge preservation following tooth extraction: a comparison between atraumatic extraction and socket seal surgery. Quintessence Int 2010; 41: 605-609.

25. Stavropoulos A, Karring ES, Kostopoulos L, Karring T. Deproteinized bovine bone and gentamicin as an adjunct to GTR in the treatment of intrabony defects: a randomized controlled clinical study. J Clin Periodontol. 2003; 30: 486-495. 